\title{
A cell-phone-based brain-computer interface for communication in daily life
}

\author{
Yu-Te Wang ${ }^{1}$, Yijun Wang ${ }^{1}$ and Tzyy-Ping Jung \\ Swartz Center for Computational Neuroscience, Institute for Neural Computational, University of \\ California, San Diego, La Jolla, CA, USA \\ E-mail: jung@sccn.ucsd.edu
}

Received 31 July 2010

Accepted for publication 13 September 2010

Published 24 March 2011

Online at stacks.iop.org/JNE/8/025018

\begin{abstract}
Moving a brain-computer interface (BCI) system from a laboratory demonstration to real-life applications still poses severe challenges to the BCI community. This study aims to integrate a mobile and wireless electroencephalogram (EEG) system and a signal-processing platform based on a cell phone into a truly wearable and wireless online BCI. Its practicality and implications in a routine $\mathrm{BCI}$ are demonstrated through the realization and testing of a steady-state visual evoked potential (SSVEP)-based BCI. This study implemented and tested online signal processing methods in both time and frequency domains for detecting SSVEPs. The results of this study showed that the performance of the proposed cell-phone-based platform was comparable, in terms of the information transfer rate, with other BCI systems using bulky commercial EEG systems and personal computers. To the best of our knowledge, this study is the first to demonstrate a truly portable, cost-effective and miniature cell-phone-based platform for online BCIs.
\end{abstract}

\section{Introduction}

Brain-computer interface (BCI) systems acquire electroencephalography (EEG) signals from the human brain and translate them into digital commands which can be recognized and processed on a computer or computers using advanced algorithms [1]. They can also provide a new interface for the users who have motor disabilities to control assistive devices such as wheelchairs.

Although EEG-based BCIs have already been studied for several decades, moving a BCI system from a laboratory demonstration to real-life applications still poses severe challenges to the BCI community [2]. To design a practical $\mathrm{BCI}$ system, the following issues need to be addressed [3-6]: (1) ease of use, (2) robustness of system performance and (3) low-cost hardware and software. In real-life applications, BCI systems should not use bulky, expensive, wired EEG acquisition devices and signal processing platforms [7]. Using these devices will not only cause discomfort and inconvenience for the users, but also affect their ability to perform routine tasks in real life. Recently, with advances

\footnotetext{
1 Author to whom any correspondence should be addressed.
}

in the biomedical sciences and electronic technologies, the development of a mobile and online $\mathrm{BCI}$ has been put on the agenda [8].

Several studies have demonstrated the use of portable devices for BCIs [7, 9]. Lin et al [7] proposed a portable BCI system that can acquire and analyze EEG signals with a custom DSP module for real-time cognitive-state monitoring. Shyu et al [9] proposed a system to combine an EEG acquisition circuit with an FPGA-based real-time signal processer. Recently, with advances in integrated circuit technology, cell phones combined with DSP [10] and built-in Bluetooth function have become very popular in the consumer market. Compared with the PC-based or customized platforms, the ubiquity, mobility and processing power of cell phones make them a potentially vital tool for creating online and portable BCIs that need real-time data transmission, signal processing and feedback presentation in real-world environments.

Although the EEG-based BCI technology using PCs and the Bluetooth transmission of bio-signals have been well established in previous studies, the feasibility of a portable cell-phone-based BCI, which supports biomedical signal acquisition and online signal processing, has never been explored. This portable system emphasizes usability 


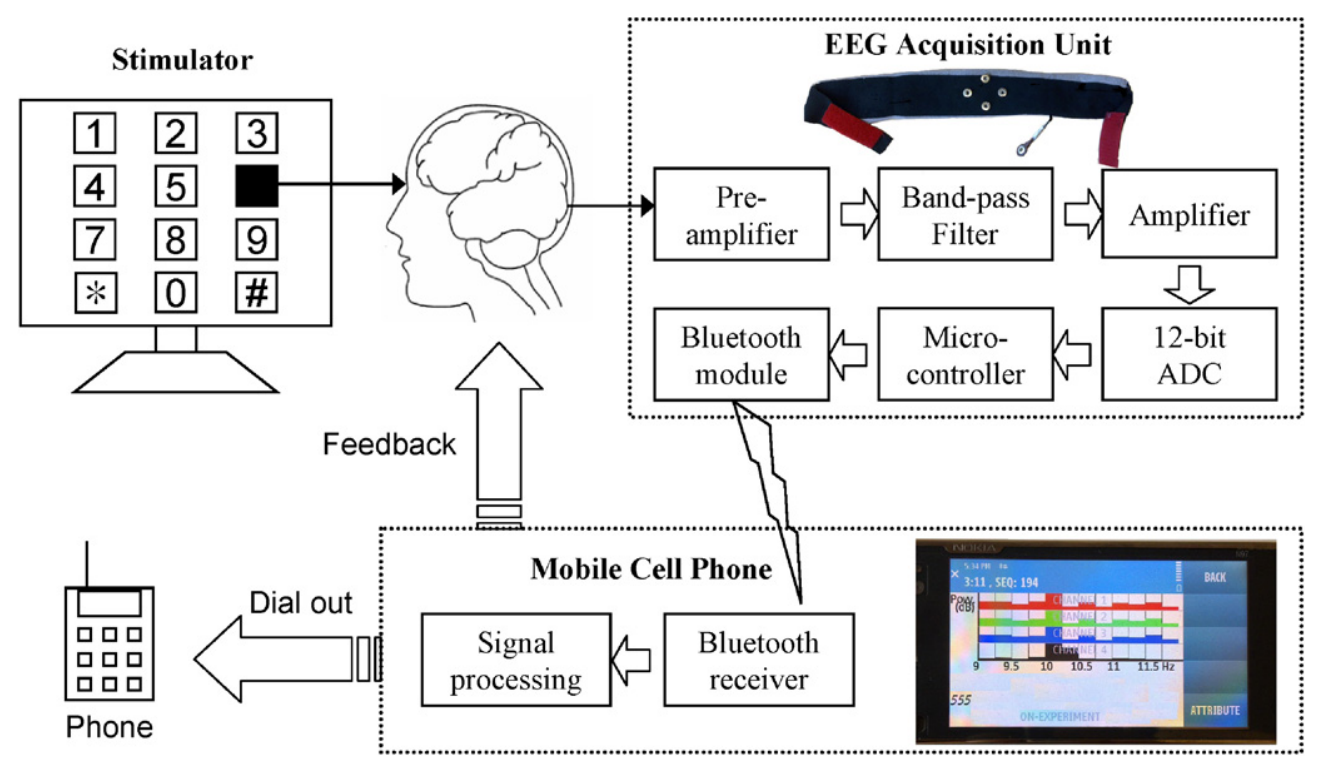

Figure 1. Diagram of the proposed system.

'on-the-go' and the freedom that cell phones enable. If a cell-phone-based BCI proves to be feasible, many current BCI demonstrations (e.g. gaming, text messaging, etc) can be realized on cell phones in practice and numerous new applications might emerge. This study integrates a portable, wireless, low-cost EEG system and a cell-phonebased signal processing platform into a truly wearable online BCI. The system consists of a four-channel bio-signal acquisition/amplification module, a wireless transmission module and a Bluetooth-enabled cell phone. The goals of this study are to demonstrate the practicality of the proposed system by specifically answering the following questions: (1) Is the quality of the EEG data collected by the custom wireless data acquisition device adequate for routine BCI use? (2) Is it feasible to implement time- and/or frequency-domain signalprocessing algorithms (e.g. EEG power spectrum estimation and EEG spatial filtering approaches) on a regular cell phone in real time?

To address these technical issues, a steady-state visual evoked potential (SSVEP)-based BCI, which has recognized advantages of ease of use, little user training and high information transfer rate (ITR), was employed as a test paradigm. SSVEP is the electrical response of the brain to the flickering visual stimulus at a repetition rate higher than $6 \mathrm{~Hz}$ [11], which is characterized by an increase in amplitude at the stimulus frequency. We adopted the widely used frequency-coding approach to build an online BCI [2, 4, 12-15] on a cell phone. In an SSVEP BCI, the attended frequency-coded targets of the user are recognized by detecting the dominant frequency of the SSVEP. To this end, several signal-processing methods have been proposed and demonstrated [16]. Among them, power spectrum density (PSD) estimation (e.g. fast Fourier transform (FFT)) is most widely used in online SSVEP BCIs [4, 12, 16]. Recently, a canonical correlation analysis (CCA) method was proposed and implemented in an online multi-channel SSVEP BCI, achieving an ITR of 58 bits $\min ^{-1}$ [17]. To explore the plausibility of an online cell-phone-based BCI platform, this study implemented and tested both single-channel FFT and multi-channel CCA methods for processing SSVEPs induced by attended targets.

\section{Method}

\subsection{System hardware diagram}

A typical VEP-based BCI using frequency coding consists of three parts: a visual stimulator, an EEG recording device and a signal-processing unit [16]. Figure 1 depicts the basic scheme of the proposed mobile and wireless BCI system. This study adapts a mobile and wireless EEG headband which is described in [8] as the EEG recording device and a Bluetooth-enabled cell phone as a signal-processing platform.

The visual stimulator comprises a 21 inch CRT monitor $(140 \mathrm{~Hz}$ refresh rate, $800 \times 600$ screen resolution) with a $4 \times 3$ stimulus matrix constituting a virtual telephone keypad which includes digits 0-9, BACKSPACE and ENTER. The stimulus frequencies ranged from 9 to $11.75 \mathrm{~Hz}$ with an interval of $0.25 \mathrm{~Hz}$ between two consecutive digits. In general, this cannot be implemented with a fixed rate of black/white flickering pattern due to a limited refresh rate of a LCD screen. Wang et al [18] recently developed a new method that approximates target frequencies of an SSVEP BCI with variable black/white reversing intervals. For example, presentation of an $11 \mathrm{~Hz}$ target stimulus on a screen refreshed at $60 \mathrm{~Hz}$ can be realized with 11 cycle black/white alternating patterns lasting (3332333233233323332332) frames in a second. Based on this approach, any stimulus frequency up to half of the refresh rate of the screen can be realized. The stimulus program was developed in Microsoft Visual C++ using the Microsoft DirectX 9.0 framework.

The EEG acquisition unit is a four-channel, wearable bio-signal acquisition unit [5]. EEG signals were amplified $(8000 \times)$ by instrumentation amplifiers, band-pass filtered 
$(0.01-50 \mathrm{~Hz})$, and digitized by analog-to-digital converters (ADC) with a 12 bit resolution. To reduce the number of wires for high-density recordings, the power, clocks and measured signals were daisy-chained from one node to another with bit-serial outputs. That is, adjacent nodes (electrodes) are connected together to (1) share the power, reference voltage and ADC clocks and (2) daisy chain the digital outputs. Next, TI MSP430 was used as a controller to digitize EEG signals using ADC via serial peripheral interface with a sampling rate of $128 \mathrm{~Hz}$. The digitized EEG signals were then transmitted to a data receiver such as a cell phone via a Bluetooth module. In this study, Bluetooth module BM0203 was used. The whole circuit was integrated into a light-weight headband.

\subsection{System software design}

The signal-processing unit was realized using a Nokia N97 (Nokia Inc.) cell phone. A J2ME program developed in Borland JBuilder2005 and Wireless Development Kit 2.2 were installed to perform online procedures including (1) displaying EEG signals in time-domain, frequency-domain and CCAdomain on the LCD screen of the cell phone, (2) band-pass filtering, (3) estimating the dominant frequencies of the VEP using FFT or CCA, (4) delivering auditory feedback to the user and (5) dialing a phone call. The resolution of the 3.5 inch touch screen of the phone is $640 \times 360$ pixels.

When the program is launched, the connection with the EEG acquisition unit is automatically established in just a few seconds. The EEG raw data are transferred, plotted and updated every second on the screen. Since the sampling rate is $128 \mathrm{~Hz}$, the screen displays about $4 \mathrm{~s}$ of data at any given time. Users can choose the format of the display between time-domain and frequency-domain. Under the frequencydomain display mode, the power spectral densities of fourchannel EEG will be plotted on the screen and updated every second. An auditory and visual feedback is presented to the user once the dominant frequency of the SSVEP is detected by the program. For example, when number 1 is detected by the system, the digit ' 1 ' is shown at the bottom of the screen and 'ONE' would be said at the same time.

Software operation and user interface include several functions. First, the program initiates a connection with the EEG acquisition unit. Second, four channels of raw EEG data are band-pass filtered at $8-20 \mathrm{~Hz}$, and then plotted on the screen every second. Third, the display can be switched to the power spectrum display or time-domain display by pressing a button at any time. Figure 1 includes a screen shot of the cell phone, which plots the EEG power across frequency bins of interest. Fourth, an FFT or CCA mode can be selected. In the FFT mode, a 512 point FFT is applied to the EEG data using a $4 \mathrm{~s}$ moving window advancing at $1 \mathrm{~s}$ steps for each channel. In the CCA mode, it uses all four channels of the EEG with a $2 \mathrm{~s}$ moving window advancing at $1 \mathrm{~s}$ steps continuously. The maximum window length is $8 \mathrm{~s}$. Detailed procedures and parameters of the CCA method can be found in [17]. To improve the reliability, a target is detected only when the same dominant frequency is detected in two consecutive windows (at time $k$ and $k+1 \mathrm{~s}, k \geqslant 4$ in the FFT mode, and $\geqslant 2$ in the
CCA mode). The subjects were instructed to shift their gaze to the next target once they heard the auditory feedback.

\subsection{BCI experiment design}

Ten volunteers with normal or corrected to normal vision participated in this experiment. All participants were asked to read and sign an informed consent form before participating in the study. The experiments were conducted in a typical office room without any electromagnetic shielding. Subjects were seated in a comfortable chair at a distance of about $60 \mathrm{~cm}$ from the screen. Four electrodes on the EEG headband were placed $2 \mathrm{~cm}$ apart, surrounding a midline occipital $(\mathrm{Oz})$ site, all referred to a forehead midline electrode (the sensor array is shown in figure 1).

The FFT- and CCA-based approaches were tested separately. All subjects participated in the experiments during which the cell phone used FFT to detect frequencies of SSVEPs, and four subjects were selected to do a comparison study between using FFT and CCA for SSVEP detection. At the beginning of the experiment, each subject was asked to gaze at some specific digits to confirm the wireless connection between the EEG headband and the cell phone. In the FFT mode, the channel with the highest signal-to-noise ratio, which is based on the power spectra of the EEG data, was selected for online target (digit) detection. Four of ten subjects who showed better performance (i.e. a higher ITR in the FFT mode) were selected to further test the CCA-based SSVEP BCI. The test session began after a couple of short practice sessions. The task was to input a ten digit phone number, 1234567890 , followed by the ENTER key to dial the number. Incorrect key detection could be erased by using the BACKSPACE key. In the CCA mode, the same task was repeated six times, leading to $11 \times 6$ selections for each subject. The EEGs in the CCA experiments were saved with feedback codes for an offline comparison study between FFT and CCA. The percentage accuracy and ITR [1] were used to evaluate the BCI performance.

\section{Results}

Tables 1 and 2 show results of the SSVEP BCI using FFT and CCA, respectively. In the FFT mode, all subjects completed the phone-dialing task with an average accuracy of $95.9 \pm 7.4 \%$ and an average time of 88.9 s. Seven of ten subjects successfully inputted 11 targets without any errors. The average ITR was $28.47 \pm 7.8$ bits $\mathrm{min}^{-1}$, which was comparable to other VEP BCIs implemented on a high-end personal computer [4]. Table 2 shows the results of the SSVEP BCI using online CCA on the cell phone. CCA achieved an averaged ITR of $45.82 \pm 2.49$ bits $\mathrm{min}^{-1}$, which is higher than that of the FFT-based online BCI of the four participants (34.22 bits $\mathrm{min}^{-1}$ ). Applying FFT to the EEG data recorded during the experiments using the online CCA resulted in an

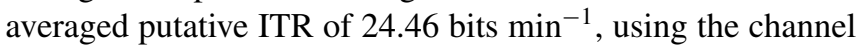
with the highest accuracy for each subject (cf right columns of table 2). 
Table 1. FFT-based online test results of the SSVEP BCI in ten subjects.

\begin{tabular}{llccl}
\hline Subject & $\begin{array}{l}\text { Input } \\
\text { length }\end{array}$ & $\begin{array}{l}\text { Time } \\
(\mathrm{s})\end{array}$ & $\begin{array}{l}\text { Accuracy } \\
(\%)\end{array}$ & $\begin{array}{l}\text { ITR (bits } \\
\mathrm{min}^{-1} \text { ) }\end{array}$ \\
\hline s1 & 11 & 72 & 100 & 32.86 \\
s2 & 11 & 72 & 100 & 32.86 \\
s3 & 19 & 164 & 78.9 & 14.67 \\
s4 & 11 & 73 & 100 & 32.4 \\
s5 & 17 & 131 & 82.4 & 17.6 \\
s6 & 11 & 67 & 100 & 35.31 \\
s7 & 11 & 72 & 100 & 32.86 \\
s8 & 13 & 93 & 92.3 & 20.41 \\
s9 & 11 & 79 & 100 & 29.95 \\
s10 & 11 & 66 & 100 & 35.85 \\
Mean & 12.6 & 88.9 & 95.9 & 28.47 \\
\hline
\end{tabular}

Table 2. CCA-based test results (ITR) of the SSVEP BCI in four subjects. In each row, the bold value highlights the maximum ITR of single channel FFT.

\begin{tabular}{|c|c|c|c|c|c|c|c|}
\hline \multirow[b]{2}{*}{ Subject } & \multirow{2}{*}{$\begin{array}{l}\text { Online } \\
\text { CCA }\end{array}$} & \multirow{2}{*}{$\begin{array}{l}\text { Online } \\
\text { FFT }\end{array}$} & \multirow{2}{*}{$\begin{array}{l}\text { Offline } \\
\text { FFT }\end{array}$} & \multicolumn{4}{|c|}{$\begin{array}{l}\text { Putative ITR from offline } \\
\text { FFT }\end{array}$} \\
\hline & & & & Ch1 & $\mathrm{Ch} 2$ & Ch3 & $\mathrm{Ch} 4$ \\
\hline s1 & 44.79 & 32.86 & 36.68 & 36.68 & 33.58 & 32.48 & 29.77 \\
\hline s2 & 46.25 & 32.86 & 26.49 & 26.49 & 10.51 & 5.91 & 9.29 \\
\hline s6 & 49.05 & 35.31 & 19.43 & 19.43 & 3.03 & 3.15 & 1.92 \\
\hline s10 & 43.18 & 35.85 & 15.24 & 2.2 & 8.46 & 15.24 & 4.21 \\
\hline Mean & 45.82 & 34.22 & 24.46 & 21.2 & 13.9 & 14.2 & 11.3 \\
\hline
\end{tabular}

\section{Discussions and conclusions}

This study designed, developed and evaluated a portable, costeffective and miniature cell-phone-based online BCI platform for communication in daily life. A mobile, lightweight, wireless and battery-powered EEG headband was used to acquire and transmit EEG data of unconstrained subjects in real-world environments. The acquired EEG data were received by a regular cell phone through Bluetooth. Advances in mobile phone technology have allowed phones to become a convenient platform for real-time processing of the EEG. The cell-phone-based platform propels the mobility, convenience and usability of online BCIs.

The practicality and implications of the proposed BCI platform were demonstrated through the high accuracy and ITR of an online SSVEP-based BCI. To explore the capacity of the cell-phone platform, two experiments were carried out using an online single-channel FFT and a multi-channel CCA algorithm. The mean ITR of the CCA mode was higher than that of the FFT approach $\left(\sim 45\right.$ bits min $^{-1}$ versus $34{\left.\text { bits } \mathrm{min}^{-1}\right)}^{-1}$ in the four participants. An offline analysis, which applied FFT to the EEG data recorded during the online CCA-based BCI experiments, showed that the target selection was less accurate using FFT than CCA, which in turn resulted in a lower ITR (table 2). The decline in accuracy and ITR in offline FFT analysis could be attributed to a lack of sufficient data for FFT to obtain accurate results. In other words, FFT, in general, required more data (longer window) than CCA to accurately estimate the dominant frequencies in SSVEPs $(6 \mathrm{~s}$ versus $4 \mathrm{~s})$.
Further, the multi-channel CCA approach eliminated the need for manually selecting the 'best' channel prior to FFT analysis.

Despite this successful demonstration of a cell-phonebased BCI, there is room for improvement. Future directions include (1) the use of dry EEG electrodes over the scalp locations covered with hairs to avoid skin preparation and the use of conductive gels, and (2) the use of higher density EEG signals to enhance the performance of the BCI [17]. However, high-density EEG might increase the computational need in BCIs. With advances in cell phone technology, more powerful onboard processors can be expected in the foreseeable future, enabling the implementation of more sophisticated algorithms for online EEG processing.

Notably, in this study, the cell phone was programmed to assess the wearer's SSVEPs for making a phone call, but it can actually be programmed to realize other BCI applications. For example, the current system can be easily converted to realize a motor imagery-based BCI by detecting EEG power perturbation of $\mathrm{mu} /$ beta rhythms over the sensorimotor areas. In essence, this study is just a demonstration of a cell-phonebased platform technology that can enable and/or facilitate numerous BCI applications in real-world environments.

\section{Acknowledgment}

This work is supported by a gift from Abraxis Bioscience, LLP. Yu-Te Wang and Tzyy-Ping Jung are supported in part by the US Army Research Laboratory and the US Army Research Office under contract/grant number W911NF-09-1-0510.

\section{References}

[1] Wolpaw J R, Birbaumer N, McFarland D J, Pfurtscheller G and Vaughan T M 2002 Brain-computer interfaces for communication and control Clin. Neurophysiol. 113 767-91

[2] Wang Y, Gao X, Hong B and Gao S 2009 Practical designs of brain-computer interfaces based on the modulation of EEG rhythms Invasive and Non-Invasive Brain-Computer Interfaces ed B Graimann and G Pfurtscheller (Berlin: Springer)

[3] Gao X, Xu D, Cheng M and Gao S 2003 A BCI-based environmental controller for the motion-disabled IEEE Trans. Neural Syst. Rehabil. Eng. 11 137-40

[4] Cheng M, Gao X R, Gao S K and Xu D F 2002 Design and implementation of a brain-computer interface with high transfer rates IEEE Trans. Biomed. Eng. 49 1181-6

[5] Lin C T, Ko L W, Chang M H, Duann J R, Chen J Y, Su T P and Jung T P 2010 Review of wireless and wearable electroencephalogram systems and brain-computer interfaces-a mini-review Gerontology 56 112-9

[6] Luo A and Sullivan T J 2010 A user-friendly SSVEP-based brain-computer interface using a time-domain classifier J. Neural Eng. 7026010

[7] Lin C T, Chen Y C, Chiu T T, Ko L W, Liang S F, Hsieh H Y, Hsu S H and Duan J R 2008 Development of wireless brain computer interface with embedded multitask scheduling and its application on real-time driver's drowsiness detection and warning IEEE Trans. Biomed. Eng. 55 1582-91

[8] Lin C T, Ko L W, Chiou J C, Duann J R, Chiu T W, Huang R S, Liang S F and Jung T P 2008 A noninvasive prosthetic platform using mobile and wireless EEG Proc. IEEE 96 1167-83 
[9] Shyu K K, Lee P L, Lee M H, Lin M H, Lai R J and Chiu Y J 2010 Development of a low-cost FPGA-based SSVEP BCI multimedia control system IEEE Trans. Biomed. Circuits Syst. 4 125-32

[10] Schneiderman R 2010 DSPs evolving in consumer electronics applications IEEE Signal Process. Mag. 27 6-10

[11] Regan D 1989 Human Brain Electrophysiology: Evoked Potentials and Evoked Magnetic Fields in Science and Medicine (New York: Elsevier)

[12] Wang Y J, Wang R P, Gao X R, Hong B and Gao S K 2006 A practical VEP-based brain-computer interface IEEE Trans. Neural Syst. Rehabil. Eng. $14234-9$

[13] Valbuena D, Cyriacks M, Friman O, Volosyak I and Graser A 2007 Brain-computer interface for high-level control of rehabilitation robotic systems Proc. 10th Int. IEEE Rehabilitation Robotics Conf. (Noordwijk: Netherlands) pp 619-25
[14] Jia C, Xu H L, Hong B, Gao X R, Zhang Z G and Gao S K 2007 A human computer interface using SSVEP-based BCI technology Proc. 3rd Int. Conf. on Foundations of Augmented Cognition (Beijing, China, July 2007) (Lecture Notes in Computer Science vol 4565) pp 113-9

[15] Muller-Putz G R and Pfurtscheller G 2008 Control of an electrical prosthesis with an SSVEP-based BCI IEEE Trans. Biomed. Eng. 55 361-4

[16] Wang Y, Gao X, Hong B, Jia C and Gao S 2008 Brain-computer interfaces based on visual evoked potentials: feasibility of practical system designs IEEE EMB Mag. 27 64-71

[17] Bin G Y, Gao X R, Yan Z, Hong B and Gao S K 2009 An online multi-channel SSVEP-based brain-computer interface using a canonical correlation analysis method J. Neural Eng. 6046002

[18] Wang Y, Wang Y T and Jung T P 2010 Visual stimulus design for high-rate SSVEP BCI Electron. Lett. 46 1057-8 